\title{
Conceptual model of the recursive entity modelling method after revision
}

\author{
Rifai Amal' ${ }^{1}$, Tzemmout Mohamed Amine ${ }^{2}$, Sadiq Abdelalime ${ }^{3}$ \\ ${ }^{1}$ Regional Center for the Professions of Education and Training (RCPET), Rabat, Morocco \\ ${ }^{2,3}$ Laboratory of Information Modelling and Communication Systems, Faculty of Sciences, University Ibn Tofail, \\ Morocco
}

\begin{tabular}{l} 
Article Info \\
\hline Article history: \\
Received Aug 11, 2020 \\
Revised Jun 1, 2021 \\
Accepted Jun 8, 2021 \\
\hline Keywords: \\
Component \\
Conception \\
Learning entity \\
Recursive entity modeling \\
method \\
Role \\
\end{tabular}

\begin{abstract}
In this work, we present the rectifications that we brought on recursive entity modeling method into lasts articles. Then, we establish the recursive entity modeling method global conceptual diagram in order to conceive a space name and database for this method in future works. Therefore, in the first section, we present an overview of the recursive entity modeling method conceptual model; then, in the second section, we present recursive entity modeling method revisited elements (relationships, component, roles, conception, path, node, connection); and in the third section, we conceive a general conceptual diagram describing the components of the recursive entity modeling method.
\end{abstract}

This is an open access article under the $\underline{C C B Y-S A}$ license.

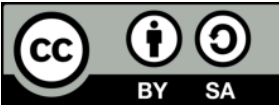

\section{Corresponding Author:}

Rifai Amal

Regional Center for the Professions of Education and Training (RCPET)

Rabat, Morocco

Email: rifaiamal10@gmail.com

\section{INTRODUCTION}

In the last decades, with the development of information and communication technologies, great masses of information are published on the web. In order to reuse, share, and organize them in distance training and e-learning frameworks, several kinds of research are achieved, and various approaches are developed. Pernin and Lejeune [1] identified two major approaches, namely: the documentalist approach and the activities-based approach. The first one, inspired from the behaviorist and cognitive teaching models [2], promotes the sharing and reuse of objects. It is based on a learning model of sourcing, referencing, and aggregating resources. It is concretized by a set of standards such as learning object metadata (LOM) [3], [4], sharable content object reference model (SCORM) [5], Normetic [6], ManUeL [7]. The second approach developed by Koper [8] puts activity at the center of the learning process and considers that knowledge objects do not constitute the key to learning success, but the activities which are associated with them; thus, leading to the learning scenario.

Several researchers in different fields of practice defined the concept of the learning scenario. In fact, in the pedagogical engineering field Paquette et al. [9] consider that the learning scenario is "the set of organized activities for learners into a coherent whole. To these activities are grafted instruments offered as support for activities (tools-inputs) and others to be made by learners (products)". In the engineering training field, Schneider et al. [10] consider that "a scenario is defined by an orchestrated sequence of phases in which learners have tasks and specific roles to play". In the technology enhanced learning (TEL) field, Daele et al. [11] have affirmed that "the learning scenario is the part of a training system that describes the 
unfolding of teaching and learning activities. This device offers to the scenario logistics and resources (technical, human, and administrative) to be implemented".

According to Pernin and Lejeune [12], the learning modeling, in the part of the second approach, consists in expressing the most precisely designed solution, with an accessible formalism, leading to a specific paradigm. This is concretized by the establishment of several languages allowing learning processes modeling such as the language (EML) [13], [14], instructional management systems learning design [15], [16], its alternative learning design language [17], the method of 4 pillars [18], and the method of Pleiades [19].

Although these languages have provided satisfactory results during their use in distance training and training frameworks, they have revealed some limitations which consist in their difficulties to:

- be easily manipulable by users,

- $\quad$ structure the educational scenarios according to different levels of hierarchical granularity,

- describe all the roles assumed by the actors,

- model collaborative and interactive learning situations, and

- $\quad$ easily express the aimed pedagogical intentions of a learning situation.

To overcome these limits, we have established a new method named "recursive entity modelling method (REMM)" [20]. This method experimented through several case studies [21]-[23], which raised some rectifications to improve its quality and facilitate its conversion into a notation language susceptible to run on computer platforms. Among these problems we cite:

- Sequence type entities can be composed of several activities to achieve an elementary objective. The fact of considering that each of these activity type entities is referenced by a set of descriptive metadata and being the subject of a pedagogically non-expressive task makes the work of modeling pedagogical scenarios very painful.

- Educational resources and roles are imprecisely described.

- There are six relationships proposed to aggregate the educational entities by REMM, namely: precedence, simultaneity, choice, hierarchy, extension, support. But, during the REMM experimentation through case studies of teaching scenarios adopting different teaching strategies (case study, problembased scenario, role-play), we noticed that we only used the first three relationships that define the physical aggregation of entities, but in some cases, we felt the need to express the pedagogical intention of the entities aggregation by using one relationship among the second three relationships more than the physical relationship.

In order to improve the REMM's quality, we brought some modifications to them in the part of several published works. In fact, in [24], we proceeded to revisit the sequence notion to support scenarists to easily model the elementary entities and to make these more semantic and expressive. Following these changes, on the one hand, we adapted the elements "Components" to take into consideration the task notion added into the sequence notion, and on the other hand, we changed the expression of the learning entity roles for making all role types and organization expression easier.

We also proposed improvements in the modeling of the components of the educational resource [25]. Our objective consisted in making REMM able to model any type of learning resource used in a scenario whatever the pedagogical intention of its employment, its role, or type. We also revised in [26], the relationships aggregating the learning scenario entities in the part of the element "Conception", to make the entities aggregation more meaningful by expressing its pedagogical intention.

In the perspective of designing a notation language based on REMM, which can be run on any computer platforms, we proceed, in this article, to present all the improvements brought on REMM to establish a general conceptual diagram allowing the conception, in a future article, of a database and a space name for our method. Therefore, in the second paragraph, we will present an overview of REMM's revisited elements. Then, in the third paragraph, we will conceive a general conceptual diagram describing the components composing REMM. In the fourth paragraph, we will end with a conclusion.

\section{OVERVIEW OF REMM'S ELEMENTS AFTER THEIR REVISION}

\subsection{Revision of entities granularity according to REMM}

Pernin and Lejeune [27] have affirmed that the granularity of a learning scenario may be at least three levels, namely: an elementary activity, a composite activity, and a structuration unit. Based on this principle, and after entity granularity revision [24], REMM considers two classes of pedagogical entities, namely: elementary and composed entities. The first ones are of two types:

- Activity: consists of the modeling of a pedagogical situation whose goal is elementarily reached by carrying out one task by one or several pedagogical roles (learner or supervisor). 
- Sequence: consists of modeling a pedagogical situation whose goal is elementarily reached by realizing several tasks assigned to roles as part of the sequence.

The composed entities are also of two types:

- $\quad$ Block: to model a pedagogical situation of an average granularity. It can be composed of other blocks and/or sequences and/or activities. Its objective is explicit, complete, and determined in terms of knowledge and skills for a specific public.

- Unit: represents the highest granularity of a pedagogical situation. It can be composed of other units and/or blocks and/or sequences and/or activities to approach a specific learning subject for a specific public. The unit can designate a course, a module, a unit of study, a semester, a license, a master, and a project.

Figure 1 illustrates the entities granularity after revision. The entity's granularity revision prompted us to rectify the elements: components, conception (that we will approach into paragraphs 2.2 (for components) and 2.5 (for conception)), to adapt these elements with the new modifications brought on entities granularities.

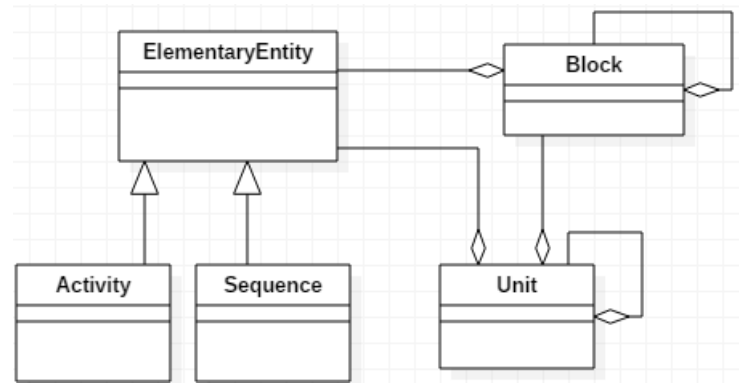

Figure 1. Educational unit class diagram [24]

\subsection{The element "components"}

The element "Components" contains entities composing the learning scenario that we name girls entities. These ones can be of composite or elementary type. The composite entities, named parent entities, can be composed by girls' entities whose granularities are less than or equal to their parent entities. Every entity girl is described as part of its entity parent, into the element Component, by the following attributes:

- $\quad$ Entity GirlReference: contains the entity girl reference in the educational scenarios database.

- Version: gives information about the version of the entity girl used in the entity. If it is not set, this attribute takes the original copy version value.

- $\quad$ Entity Girl Key: used to attribute the second entity identifier to the entity girl. This one will be used to address it into the parent entity conception (design).

As well as the entities of the type of sequence, these can be in turn composed of more than one task designated by element "Task" and described by the following attributes:

- TaskRef: references the task as part of the educational entity.

- TaskStatut: shows if the task has a main or complementary status or if it is a logistical task type whose purpose is to support the implementation of other tasks in the educational scenario.

- $\quad$ Task description: describes the task to perform by the role with sentences.

Figure 2 clearly shows the addition of the element tasks as an item of the element "Components":

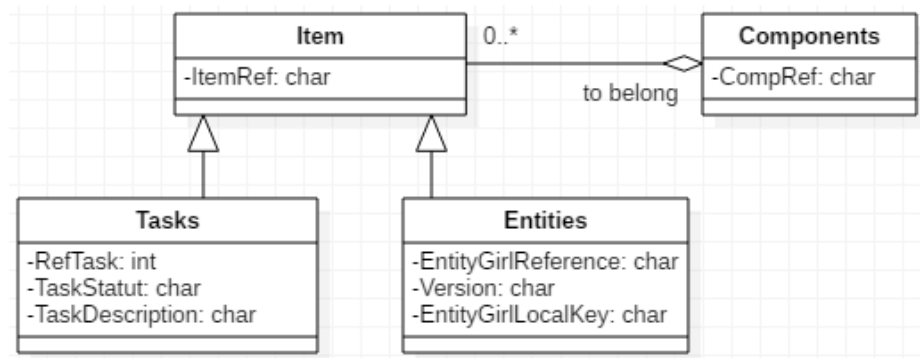

Figure 2. Element "components" as revisited into [24] 


\subsection{Learning entities relationships}

To form a composite entity, its entity's girls must be aggregated with relationships. For this, the revised REMM version [26] classified the six types of relationships into two categories, namely:

a. Physical relationships informing about the physical position of an educational entity vis-à-vis others within the learning scenario. These physicals relationships are:

- Precedence: permits to execute the entities in a sequential way,

- Simultaneity: allows realizing several entities at the same time, and

- Choice: allows choosing the entities to realize according to specific conditions related to the previous learning results or other circumstances.

b. Pedagogical relationships expressing the pedagogical intention of introduction of an element (Entity_unique, Task, Path, or Node) in the scenario concerning another element (Entity_unique, Task, Path, or Node). These relationships are:

- Hierarchy: expresses the case when a learning entity is described in detail by another one,

- Extension: allows superimposing several learning scenarios whose execution is conditional, and

- Accompaniment: permits expressing the assistance situations to learner works such as feedback and regulation situations of the accomplished evaluations, of the real learning situations).

\subsection{Educational situation roles according to REMM}

The roles of a learning scenario can be realized by a learner or staff. The role modeling as its revised version [24] is carried out by the following elements:

a. Individual: can be learner or staff, who can be arranged as part of a group, subgroup or each one works independently. Each individual is referenced by IndividuRef and has a set of attributes, which identify him such as first name, and last name.

b. Group: represents the organization of individuals (learners and/or staff assuming roles in the educational scenario). This element contains the following attributes:

- $\quad$ Groupe Ref: references the group with a single value.

- Groupe Title: it is optional; it allows assigning names to groups.

- Members Work Type: it can take one of the following values: Common, different to show whether group members have the same task to achieve, or each one has its task.

- Members Work Strategy: to show whether group members collaborate, cooperate or each one works individually to realize one or set of activities in the entity.

c. Role: permits to describe the role assumed by a group or an individual. this property has the following attributes:

- $\quad$ RoleRef: References the role

- $\quad$ RoleTitle: designates the title assigned to the actors (learner and/or staff).

d. Task: describes the work to be performed by roles; this attribute accepts as value the task reference already defined into the element "Components".

Figure 3 illustrates, the pedagogical situation roles modeling by their assigning tasks to do and defines the various pedagogical entities granularities according to REMM.

\subsection{The component "Conception": educational situation scenario according to REMM}

As defined in [26], the component "Conception" can be a single entity, a path, a node, or a task (the last case for modeling the elementary entities Activity and Sequence).

\subsection{1. "Path" component}

The component "Path" allows executing sequentially two or more elements. The element "Path" is modeled by two elements. The first is PathRef, which is to reference the "Path" by a unique value at the level of the educational entity. The second one is "Connection" (one or more). Each connection is referenced by ConnRef, Element, and Relationship.

A ConnRef allows referencing the connection into the parent entity. An element can either be "Entity_Unique", "Task", "Path" or "Node". So, this component is identified by the following attributes.

a. ElementRef: allows referencing the element within its parent entity.

b. ElementType: permits to define the type of the element. It can take one value amongst these ones: Task, Entity_unique, Path, or Node.

Relationship defines the physical and the pedagogical relationship aggregating the elements of the component "Connection". So, it is composed of the following attributes and sub-components.

a. RsRef: permits to reference the Relationship by a unique value.

b. SourceElementRef: it represents the first performed element into the relationship component regarding PhysicalRelationship sense. 


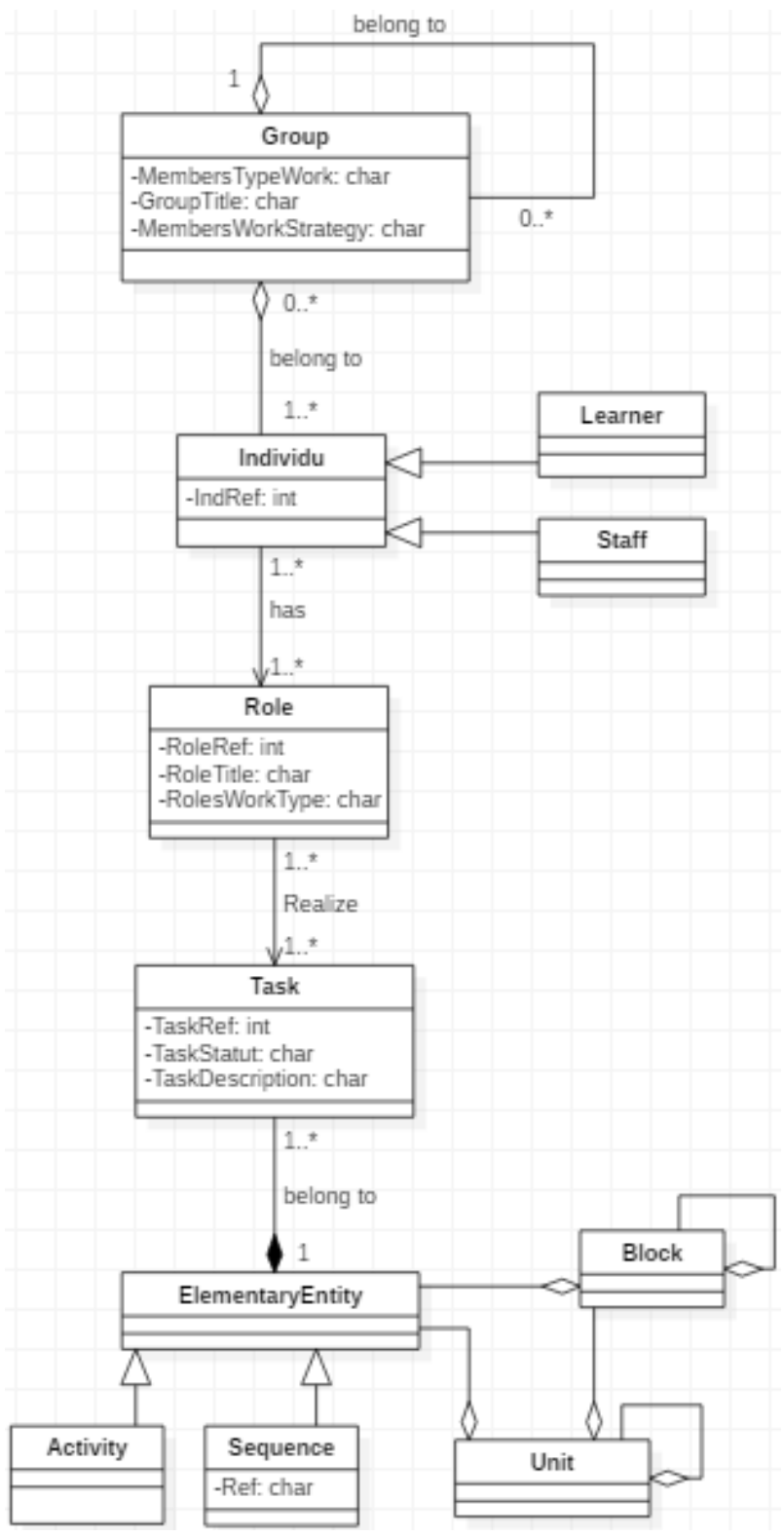

Figure 3. Conceptual diagram of roles and their tasks modeling [24]

c. TargetElementRef: it represents the second performed element into the relationship component regarding PhysicalRelationship sense.

d. PhysicalRelationship:

- PhysicalRelationshipValue: allows to aggregate the source and the target elements by the relationship of "Precedence" or "ComplexRelationship". This second value of relationship expresses the case, where one or the two elements of component "Connection" are of "Node" type, and the elements corresponding to grains of the node are aggregated with the other elements of the component Connection (which is outside (up/downstream) of the node) with a relationship different to precedence; by taking in consideration that we precise the physical relationship between node grains elements and those situated outside when we describe node grains elements.

- PhyRelSCondition: represents the condition of the validation of the PhysicalRelationship (the validation of the element defined into SourceElementRef to execute the element referenced into TargetElementRef).

e. PedagogicalRelationship: allows informing about the pedagogical relationship between the elements corresponding to the component "Connection" by specifying the educational role played by an element vis-a-vis the other one. It is composed of the following attributes: 
- PedagogicalRelationshipValue: it can take one of the values: "Undefined" (if the two elements haven't connected by a pedagogical relationship), "Hierarchy", "Extension", "Accompaniment" (their definitions are already introduced in the previous paragraph), or "ComplexRelationship" (if one or two elements into "Connection" are of "Node" type; and the element corresponding to each grain of the node is aggregated with the element which is outside (up/downstream) of the node with a different relationship).

- $\quad$ RsDir: permits to inform about the source and the target element regarding Pedagogical Relationship. It can take as value: Normal (if the source element and the target one is those defined respectively by SourceElementRef and TargetElementRef regarding PedagogicalRelationship) and Reverse (if the element referenced into TargetElementRef is the source element and the element referenced into SourceElementRef is the target element regarding PedagogicalRelationship).

- PedRelSCondition: expresses the condition of execution of the pedagogical relationship.

Figure 4 illustrates the rectified component "Path" taking into account changes made into relationships.

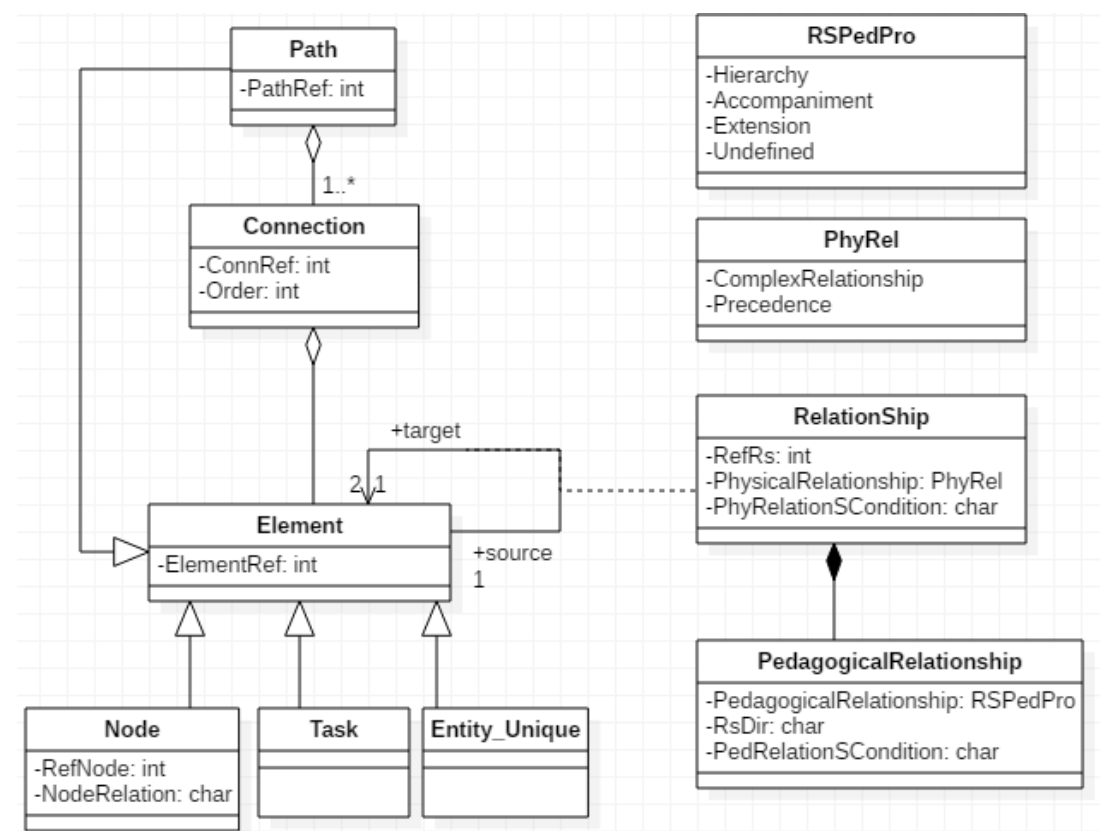

Figure 4. Class diagram illustrating the rectified component "Path" [26]

\subsubsection{Node}

The component "Node" plays the role of a control element expressing the two cases, which are selection of an element among several others and execution of several elements in a parallel way. Consequently, it is composed of three attributes and components; they are NodeRef, NodeRelationshipType, and Grain.

NodeRef allows referencing the node within the parent entity. NodeRelationshipType: takes as value the type of the physical relationship aggregating the elements attributed to the grains of the node. So, it can take one of the two values:

a. Simultaneity: corresponds to the case of the parallel execution of the elements of the node grains.

b. Choice: corresponds to the case of the selection of the element which is executed amongst those defined into the node grains according to a condition.

Grain (two or more), whose attributes and components are:

a. GrainRef: allows referencing the grain within the parent entity.

b. OpeningCondition: indicates the condition permitting the execution of the element of the grain.

c. Element: represents the element to execute. It is composed of the following attributes.

- $\quad$ ElementRef: allows referencing the element within its parent entity.

- ElementType: permits the definition of the type of the element. It can take one value amongst the following values: Task, Entity_unique, Path, or Node.

We note that the element corresponding to each grain of the node can be related with the elements corresponding to others node grains by a pedagogical relationship (by considering that the physical 
relationship is already defined by the attribute "NodeRelationshipType"), and with the elements situated into the outside of the node by a physical and pedagogical relationship. Therefore, the component "Grain" is composed also of the sub-components "PedagogicalRelationship" to express the pedagogical relationship between elements of the node grains and "Relationship" for expressing the relationship between the elements of the node grains and those situated outside of the node. These sub-components are detailed in the following.

PedagogicalRelationship allows informing about the pedagogical relationships between the current element and the others corresponding to grains of the node. It is composed of one or several items depending on whether there are pedagogical relationships between them, otherwise this property defaults to none. The attributes of the sub-element item are:

a. PedagogicalRelationshipValue: it represents the value of the pedagogical relationship which can take

"Undefined" if two elements are not connected by a pedagogical relationship, or "Hierarchy", "Extension", "Accompaniment", defined in subsection 2.3.

b. SourceElementRef: it represents the first element of the pedagogical relationship.

c. TargetElementRef: it represents the element of pedagogical relationship that represents the property defined into PedagogicalRelationshipValue vis-a-vis the element defined into SourceElementRef. Example: considering SourceElementRef=Activity0, TargetElementRef=Sequence1.1 and PedagogicalRelationshipValue=hierarchy; this means that Sequence1.1 is an entity that proposes a detailed work approaching the same subject of Activity0

d. PedRelSCondition: expresses the condition of the pedagogical relationship execution. Example: we take again the previous example, so, we consider that the execution of Sequence1.1 is achieved according to a condition that we specify into the attribute PedRelSCondition.

Relationship allows defining the physical and pedagogical relationship between the current element and those situated outside of the node that can be upstream and/or downstream. So, this component is composed of two attributes and sub-components. The first one is RsRef, which permits to reference the component "Relationship" by a unique value. The second one is Item, which represents an item of the relationship, it takes as attributes:

a. Position: defines the position of the element with which the grain element will be aggregated.

b. PhysicalRelationship: takes as value the physical relationship that aggregates the grain element and the element situated outside of the node. These values are "Precedence", "Choice", and "Simultaneity".

c. SourceElementRef: it represents the first element into the relationship component regarding PhysicalRelationship sense.

d. TargetElementRef: it represents the second element into the relationship component regarding PhysicalRelationship sense.

e. PhyRelSCondition: expresses the condition of execution of the physical relationship.

f. PedagogicalRelationship: allows informing about the educational relationship between the node grain element and that situated outside of the node. So, it is composed of the following attributes:

- PedagogicalRelationshipValue, which can take either "Undefined" (if the two elements have not connected by a pedagogical relationship) or "Hierarchy", "Extension", "Accompaniment", which defined in subsection 2.3,

- RsDir, which permits to inform about the source and the target element regarding PedagogicalRelationship. It can take one of the values, which are Normal (if the source element and the target element are referenced respectively by SourceElementRef and TargetElementRef) and Reverse (if the element referenced by TargetElementRef is the source element and the element referenced by SourceElementRef is the target element regarding pedagogical relationship [the target element is that which represents the PedagogicalRelationshipValue property vis-a-vis the source element]).

- PedRelSCondition: expresses the condition of the pedagogical relationship execution. Example: Same example proposed for a physical relationship.

In Figure 5, we present the class diagram of the rectified component "Node" including the REMM's relationships new changes. Figure 6 illustrates the class diagram of the rectified component "Conception" including the changes brought on elements path and node.

\subsection{Improvement of the learning resources modeling}

To consider the pedagogical intention of use of educational resources, their types, and the fact that they are provided by the staff or produced by the learner, Rifai et al. [25] propose to define it in the component "Learning-Object" which can be one of different types.

a. Knowledge: it can be a part of a course or a chapter, exercise, diagnostic assessment, formative evaluation, summative evaluation, a correction of an evaluation, correction of an exercise, a consign, a 
report, a book, a roman, a reference to a page into a book, a message, a message attachment. It can take the different format: text, image, video...

b. Tools: represents the Materials and/or software that can be used in the educational situation.

c. Services: represents the services used to carry out the educational entity, such as videoconference, email service, search-index, and digital platform (forum, social site, frequently asked questions, classroom).

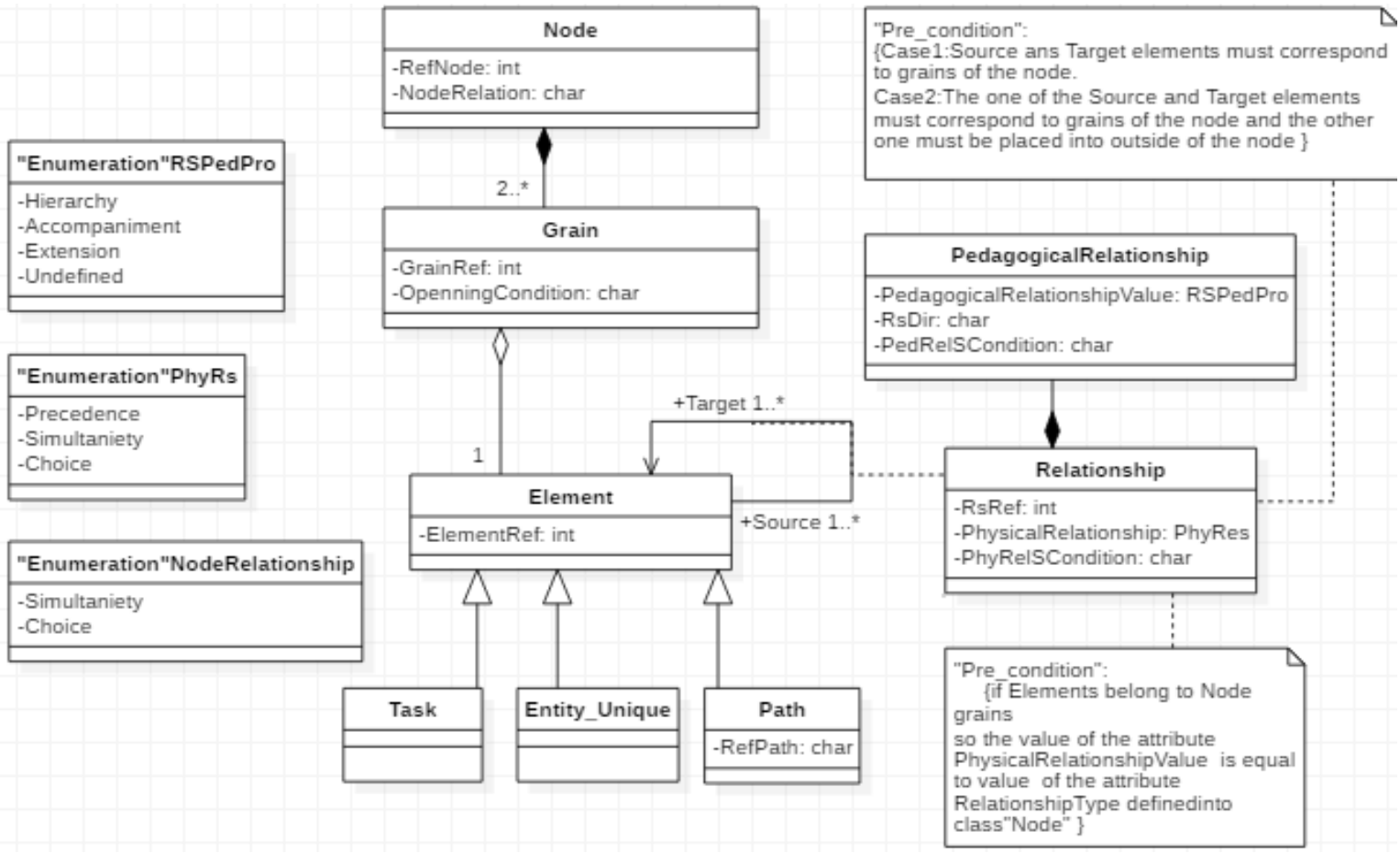

Figure 5. Class diagram illustrating the rectified component "Node" [26]

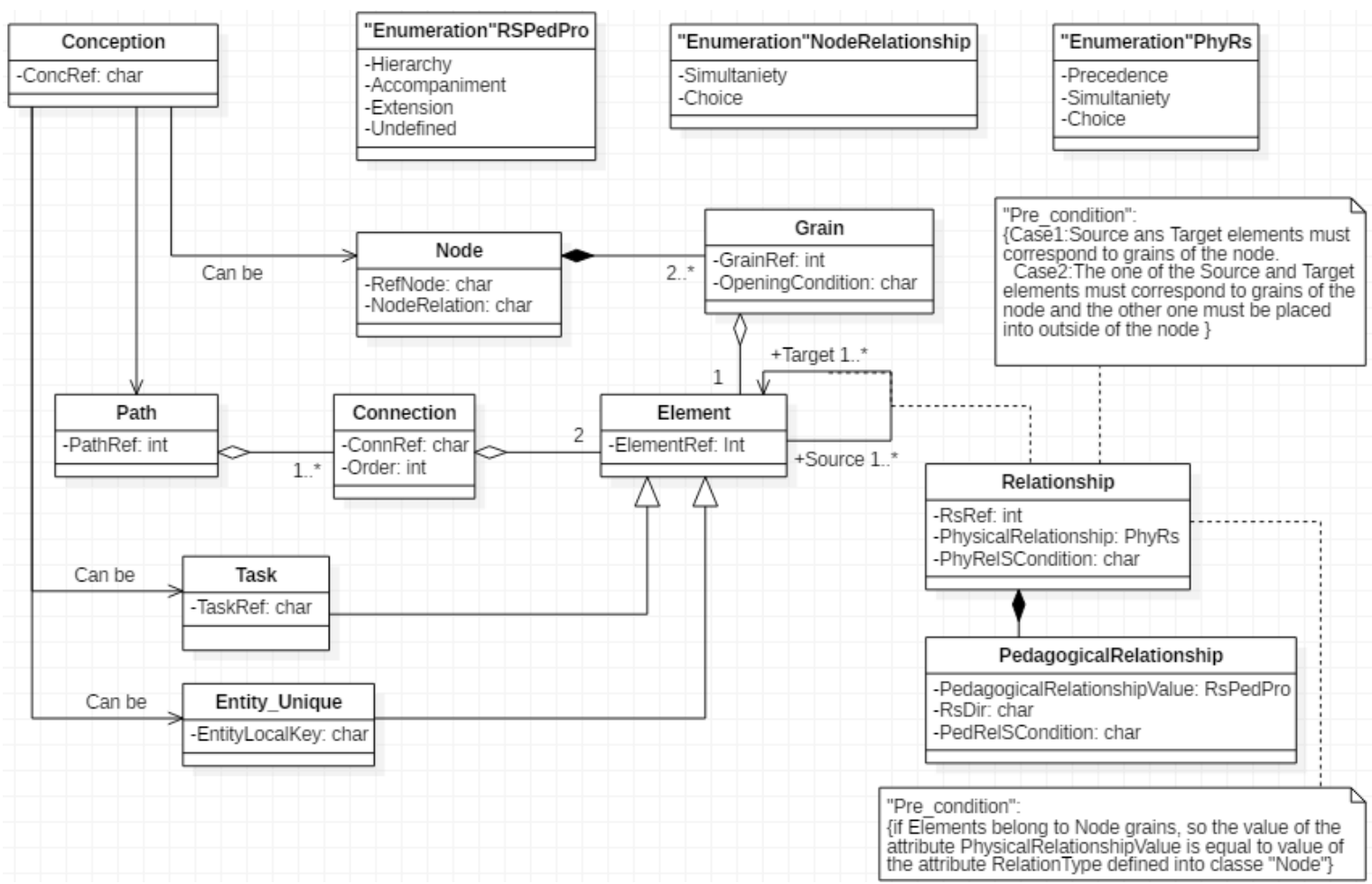

Figure 6. Class diagram illustrating the rectified component "Conception" [26] 
To correctly describe the learning object, the improved component "Learning-Object" contains the following attributes.

a. LORef: it is unique and represents the learning object reference into the educational entity.

b. PedagogicalType: allows classifying learning objects into four categories regarding whether the learning object is deployed into learning or it has resulted after the realization of an entity, as well as the pedagogical intention of its use. Thus, this attribute can take one of the following values:

- Learning and support elements: represents knowledge (courses, exercises, documents, books, poems), services (videoconference, forum, e-mail) and tools (hardware and software) on which bears the educational entity.

- Schedule support elements: represents knowledge (video, images, books, encyclopedias), tools (hardware, software), and services offered in addition to the learning and support elements to support learners to achieve their works and to diversify teaching methods and means.

- $\quad$ Produced element: represents the outcomes of learning entity (knowledge, tools, and services produced after the educational entity achievement).

- Communication element: represents the notifications and synchronous or asynchronous messages communicated between the roles of entities.

c. Title: represents the title of the deployed knowledge, the name of the tools (software or hardware), the title of the used services (conferencing, digital platform, the engine of search), and the message subject.

d. Archiving format: allows to inform about the learning resource format (digital or no-digital)

e. Reference: This attribute includes the following properties:

- Description: describes the resource (book publisher, year of publication, authors), tool (digital camera or image processing software), and service (discussion forum, asynchronous multi-point conference). In this work, we do not address in detail the description of each type of learning resources.

- $\quad$ URL: contains the web address of a numeric object.

Figure 7 shows the class diagram of the revised REMM's learning objects.

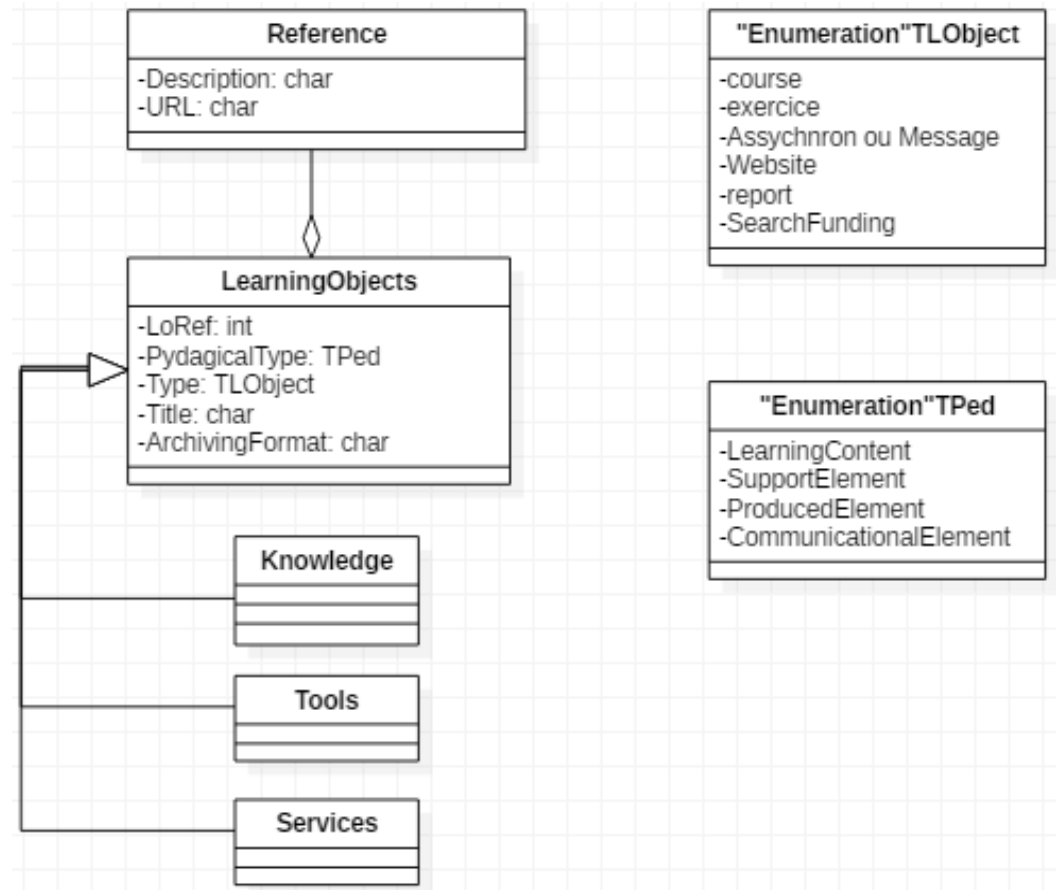

Figure 7. Conceptual diagram of learning object after revision [25]

\section{GLOBAL CONCEPTUAL DIAGRAM OF REMM}

Basing on the above-mentioned diagrams of the revised components of the recursive entity modeling method, we conclude in Figure 8 the general conceptual diagram of this method. 


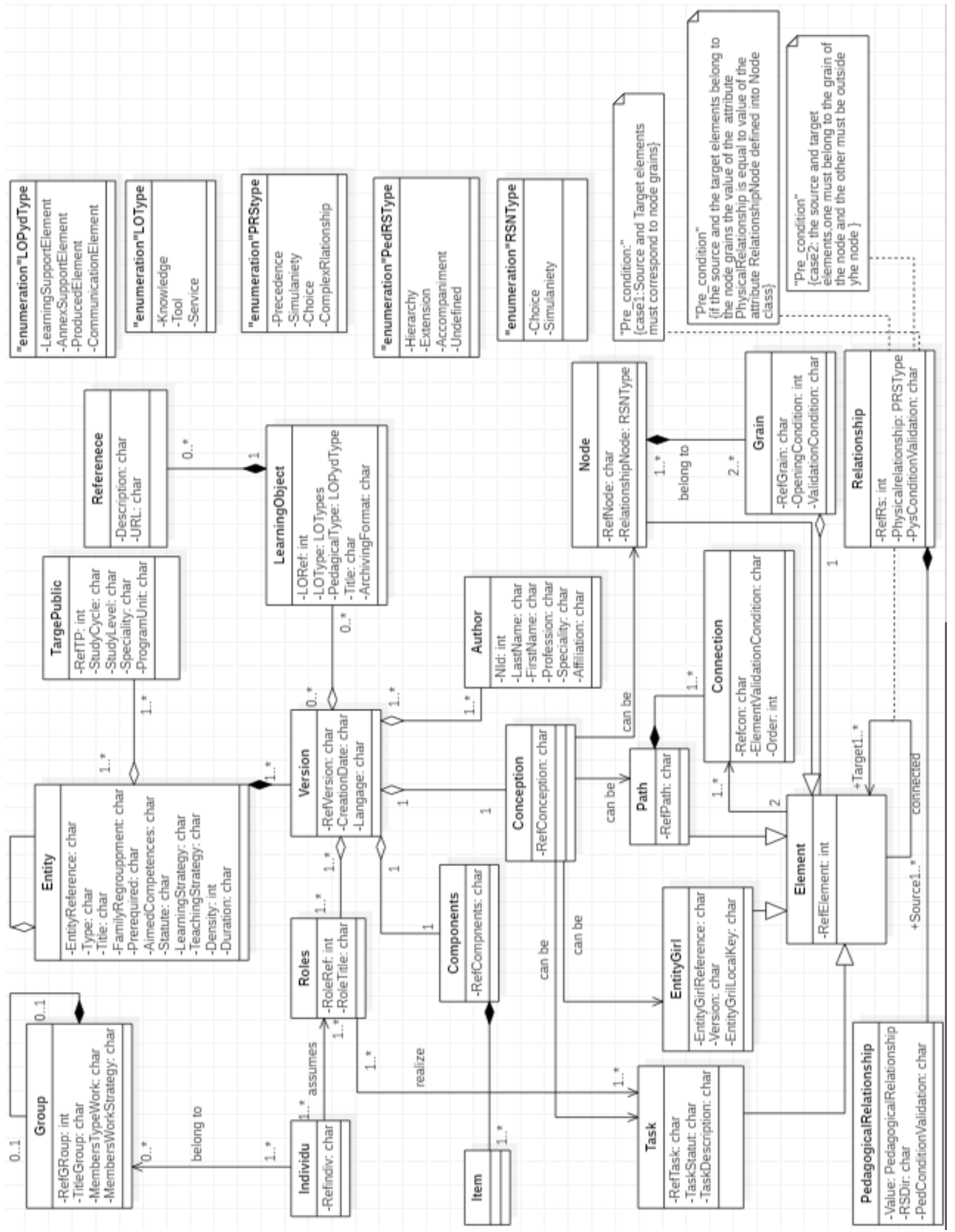

Figure 8. Global conceptual diagram of REMM

\section{CONCLUSION}

In this work, we presented a new revised version of the recursive entity modeling method (REMM). In fact, in the last publications, we published the revised REMM components, and by basing on those revisions, we conceived, in this work, a REMM's global conceptual diagram in order to facilitate the conception of REMM's database in future work. So, in the second paragraph, we approached all revised REMM components. Then, in the third paragraph, we conceived a general conceptual diagram for our method REMM after its revision. Afterward, in the fourth paragraph, we presented a conclusion. 


\section{REFERENCES}

[1] J. Pernin and A. Lejeune, "Model for the reuse of learning scenarios (in French: Modèle pour la réutilisation de scénarios d'apprentissage)," in TICE Méditerranée, 2004.

[2] P. A. Caron, "Model-driven engineering for the construction of educational devices on training platforms (in French: Ingénierie dirigée par les modèles pour la construction de dispositifs pédagogiques sur des plateformes de formation)," Doctoral Thesis, Université des Sciences et Technologies de Lille, France, 2007.

[3] "IEEE Learning Technology Standards Committee (LTSC) Systems Interoperability in Education and Training," 2002. [Online]. Available: http://ltsc.ieee.org/wg12/doc.html.

[4] B. de la Passardière and M. Grandbastien, "Presentation of the LOM v1.0, IEEE standard (in French: Présentation de LOM v1.0, standard IEEE," Revue Sciences et techniques éducatives Hors série, pp. 211-218, 2003.

[5] "SCORM "ADL Sharable Content Object Reference Model Version 1.3, Working draft 0.9," 2002. Available: https://scorm.com.

[6] "Standardized description of resources: towards an educational heritage NORMETIC, version 1.0 (in French: La description normalisée des ressources: vers un patrimoine éducatif NORMETIC, version 1.0)," CREPUQ, Novasys inc., Montréal, Canada, 2003.

[7] B. de LaPassadière and P. Jarraud, "ManUeL, a LOM application profile for C @ mpuSciences (in French: ManUeL, un profil d'application du LOM pour)," Sticef, vol. 11, p. 1-39, 2004

[8] R. Koper, "From change to renewal: Educational technology foundations of electronic learning environments," Educational Technology Expertise Center, Netherlands: Open University of the Netherlands, 2000. Available: https://www.academia.edu/10869338/From_Change_to_Renewal_Educational_Technology_Foundations_of_Electr onic_Learning_Environments

[9] G. Paquette, F. Crevier, and C. Aubin, "Engineering method of a learning system (MISA) (in French: Méthode d'ingénierie d'un système d'apprentissage (MISA))," Revue informations in cognito, no. 8, 1997.

[10] D. Schneider, "Design and implementation of rich educational scenarios with community portals (in French: Conception et implémentation de scénarios pédagogiques riches avec des portails communautaires)," in Second colloque de Guéret, 2003.

[11] A. Daele, C. Brassard, L. Esnault, M. Donoghue, E. Uytterbrouk, and R. Zeiliger, "Design, implementation, analysis and evaluation of pedagogical scenarios using ICT (in French: Conception, mise en œuvre, analyse et évaluation des scénarios pédagogiques recourant à l'usage des TIC)," in Rapport du projet Recre@sup-WP2 FUNDP, 2002

[12] J.-P. Pernin and A. Lejeune, "IMS Learning Design: a language for notation, modeling, or design? (in French: IMS Learning Design: langage de notation, de modélisation ou de conception?)," in Atelier sur IMS LD, Paris, 2005.

[13] "EML, Educational Technology Expertise Centre," 2001. [Online]. Available: http://eml.ou.nl/introduction/articles.htm.

[14] R. Koper, "Modeling units of study from a pedagogical perspective: the pedagogical metamodel behind EML," Educational Technology Expertise Centre, Open University of the Netherlands, 2001. Available: https://eml.ou.nl/introduction/articles.htm.

[15] "Learning Design Specification," Learning Design Specification | IMS Global Learning Consortium. [Online]. Available: https://www.imsglobal.org/learningdesign/. [Retrieved: 14-September-2009].

[16] A. Lejeune, "IMS Learning Design," Distances et savoirs, vol. 2, no. 4, pp. 409-450, 2004, doi: 10.3166/ds.2.409450.

[17] C. Martel, "Modeling of joint activities: Roles, places and positions of participants (in French: La modélisation des activités conjointes. Rôles, places et positions des participants)," Thesis, Université de Savoie, 1998.

[18] C. Martel, A. Lejeune, C. Ferraris, and L. Vignollet, "Scripting the 4 pillars of pedagogy (In French: Scénariser les 4 piliers de la pédagogie)," in Colloque EIAH'07, Lausanne, 2007.

[19] E. Villiot-Leclercq, "Modèle de soutien à l'élaboration et à la réutilisation de scénarios pédagogiques (in French: Support model for the development and reuse of educational scenarios)," Ph.D. thesis, Université Joseph-Fourier, France, 2007.

[20] R. Amal and R. Messoussi, "Modelling method of recursive entity," British Journal of Educational Technology, vol. 43, no. 1, pp. 28-38, 2010, https://doi.org/10.1111/j.1467-8535.2010.01141.x.

[21] A. Rifai, R. Messoussi, and M. Bakrim, "Learning scenario modeling by the recursive entity modeling method: A case study," in International Conference on Models of Information and Communication Systems (MICS'10), Rabat, 2010, https://doi.org/10.1145/3090354.3090454.

[22] A. Rifai, M. Bakrim, and A. Sadiq, "Modeling of Learning Scenario adopting Case Study Strategy by Recursive Entity Modeling Method," in 2016 4th IEEE International Colloquium on Information Science and Technology (CiSt), Tangier, Morocco, 2016, pp. 521-526, doi: 10.1109/CIST.2016.7805104.

[23] A. Rifai, M. Joutey Tahri, M. Bakrim, and A. Sadiq, "Modeling of Problem Based Learning Strategy by Recursive Entity Modeling Method," 2nd International Conference on Big Data, Cloud and Applications (BDCA'17), Tetuan, 2017, doi: 10.1145/3090354.3090454. https://doi.org/10.1145/3090354.3090454

[24] A. Rifai, M. Bakrim, and M. Dahchour, "Revision of Recursive Entity Modeling Method: Notion of Sequence, Roles and "Components" and "Conception" Elements," International Journal of Advanced Research in Computer Science and Software Engineering, vol. 5, no. 5, May 2015.

[25] R. Amal, T. J. Majda, B. Mhamed, and S. Abdelalim, "Revision of the learning resources modeling concept by Recursive Entity Modeling Method (REMM)," Proceedings of the 2nd international Conference on Big Data, Cloud and Applications, Tetuan, no. 90, 2017, pp. 1-6, doi: 10.1145/3090354.3090446. 
[26] A. Rifai, M. Bakrim, R. Messoussi, and R. Touahni, "Amelioration of the relationships aggregating educational entities in the Recursive entity modeling method," International Journal of Applied Engineering Research, vol. 11, no. 24, pp. 11967-11975, 2017

[27] A. Lejeune and J-P. Pernin, "A taxonomy for scenario-based engineering," in Cognition and Exploratory Learning in Digital Age (CELDA 2004) Proceedings, Lisboa, Portugal, 2004, pp. 249-256.

\section{BIOGRAPHIES OF AUTHORS}
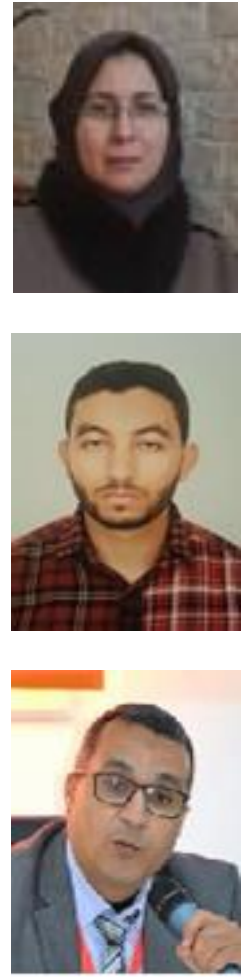

Rifai Amal is an associate professor of Computer Science at Regional Center for the Professions of Education and Training (RCPET), Rabat, Morocco. She is also the head of the Team of Research in Engineering of Computing Environment for Human Learning.

Tzemmout Mohamed Amine is a professor of qualifying secondary education (Souss Massa Academy) and a research student at Department of Computer Science, Sciences Faculty, Ibn Tofail University (Kenitra Morocco).

Abdelalim Sadiq is an associate professor of Computer Science, Sciences Faculty, Ibn Tofail University (Kenitra Morocco) and the head of SIM TEAM at MISC Laboratory. 